\title{
HIERARCHICALLY COUPLED ULTRADIAN OSCILLATORS GENERATING ROBUST CIRCADIAN RHYTHMS
}

- RAFAEL A. BARRIO

Instituto de Física, UNAM, Apartado Postal 20-364, 01000 México, D.F., Mexico

(E.mail: barrio@anatolia.ifisicacu.unam.mx)

- LIMEI ZHANG

Departamento de Fisiología, Facultad de Medicina, UNAM, 04510 México, D.F., Mexico

\section{- PHILIP K. MAINI}

Centre for Mathematical Biology, Mathematical Institute, University of Oxford, 24-29 St. Giles, Oxford OX1 3LB, United Kingdom

\begin{abstract}
Ensembles of mutually coupled ultradian cellular oscillators have been proposed by a number of authors to explain the generation of circadian rhythms in mammals. Most mathematical models using many coupled oscillators predict that the output period should vary as the square root of the number of participating units, thus being inconsistent with the well-established experimental result that ablation of substantial parts of the suprachiasmatic nuclei (SCN), the main circadian pacemaker in mammals, does not eliminate the overt circadian functions, which show no changes in the phases or periods of the rhythms. From these observations, we have developed a theoretical model that exhibits the robustness of the circadian clock to changes in the number of cells in the SCN, and that is readily adaptable to include the successful features of other known models of circadian regulation, such as the phase response curves and light resetting of the phase. (C) 1997 Society for Mathematical Biology
\end{abstract}

1. Introduction. Circadian rhythms provide a ubiquitous adaptation of most eukaryotes and some prokaryotes to the environmental day and night alteration, and they express the working of a mysterious endogenous oscillator, a clock-like mechanism that provides temporal organization. This clock-like mechanism (circadian pacemaker) functions autonomously but can be entrained by environmental cyclic changes. 
There have been great advances in the research of the clock mechanism during this decade. Recent molecular studies, extensively performed on fruit fly Drosophila melanogaster and bread mold Neurospora crassa suggest that the regulation of gene expression may lie at the heart of the clock mechanism (Hardin et al., 1990; Edery et al., 1994; Sehgal et al., 1995; Gekakis et al., 1995; Myers et al., 1996; Lee et al., 1996). In the fruit fly, two genes, per (for period, Konopka and Benzer, 1971) and tim (timeless, Sehgal et al., 1994) are essential components of the circadian clock. Mutations in either of these genes can produce arrythmicity or change the period of the rhythm by several hours. The picture of how the per and tim proteins interact is far from complete, although it seems that they work as a team to generate an oscillating cycle of activity in their genes and probably other genes, which in turn set up daily rhythms in the fly's physiology and activity (Sehgal et al., 1995; Vosshall et al., 1994).

In mammals, the main circadian pacemaker is located in the suprachiasmatic nuclei (SCN) of the anterior hyphothalamus (for reviews, see Meijer and Rietveld, 1989; Moore, 1992). Disruption of SCN function by lesioning or surgical isolation abolishes circadian rythmicity, which can be restored by transplantation of fetal tissue into the third ventricle of SCN-lesioned hosts (Drucker-Colin et al., 1984; Lehman et al., 1987; Ralph et al., 1990). The autonomous nature of the SCN pacemaker activity has been demonstrated by evidences that the circadian rhythm in electrical activity persists when the SCN are isolated surgically from the rest of the hypothalamus (Inouye and Kawamura, 1979) or isolated in vitro (Gillette, 1991; Prosser et al., 1990; Zhang and Aguilar-Roblero, 1995). However, what composes the clock and how it is organized are currently matters of discussion. At present two possibilities are apparent: either there is a class of pacemaker cells capable of sustaining a 24-hour oscillation individually in its own activity, or the circadian pacemaker is an emergent property of circuit interactions within the SCN. In this paper, we explore the latter possibility and shall discuss the former one at the end.

Several mathematical models aiming to explain the circadian pacemaker function have been based on coupled cellular oscillators (Winfree, 1967; Enright, 1980; Pittendrigh, 1974; Pavlidis, 1969, 1975). These interacting cellular oscillators are thought to function by neuronal communication mediated by neurotransmitter release. However, the validity of these models has been jeopardized by a series of experiments consisting of the administration of tetrodotoxin (TTX) (see, for example, Schwartz et al., 1987; Earnest et al., 1991; Newman et al., 1992). TTX can successfully block sodium-dependent action potentials, and therefore block the neurotransmitter release, but cannot alter the precision of the time-keeping machine.

These observations suggest that the classical sodium-dependent axonal neurotransmission may not be needed for the time-keeping machine. Thus, the biological basis on which previous mathematical models are based is 
called into question. The ways in which an ultradian oscillation in the cellular level can induce a circadian rhythm is by no means clear, either experimentally or theoretically. However, recent findings suggest that the coupled oscillators idea cannot be ruled out.

Ultradian $\mathrm{Ca}^{2+}$ oscillations were recently found (Van den Pol et al., 1992) in both neurons and glia cultured from SCN cells. The phase and period of glia $\mathrm{Ca}^{2+}$ oscillations are affected by several neurotransmitters, but cannot be blocked by TTX. Astrocytes show very regular rhythms of cytoplasmic $\mathrm{Ca}^{2+}$ concentration with periods ranging from 7 to $20 \mathrm{sec}$. The oscillating wave travels from one glia cell to another through gap junctions. The intercellular wave can increase the period of the independently oscillating glia cells. The intracellular $\mathrm{Ca}^{2+}$ signalling in glial networks can discriminate the nature of the stimulus and possibly enable glial cells to perform long distance signalling. Moreover, this network can be modulated by activation of neuro-transmitter receptors, by nerve electrical activity, or even by the increase in $\left[\mathrm{Ca}^{2+}\right]$ itself. $\mathrm{Ca}^{2+}$ signals in glia can feed back on neurons (for review see Verkhratsky and Kettenmann, 1996).

Van den Pol et al. (1992) proposed that the glia $\mathrm{Ca}^{2+}$ oscillations could be the basis of the coupled oscillators generating the circadian signals. This opens a new perspective on the research of the clock machinery. Mathematical modelling has suggested that high-frequency oscillators with periods within the same range as those shown in Van den Pol's paper may bc the basis for a low-frequency oscillator such as a circadian oscillator (Pavlidis, 1969; Winfree, 1975). However, there is an important feature that cannot be explained by the existing coupled oscillator models: the relative scale invariance of the circadian oscillator.

There are two SCN located at the anterior hypothalamus. It is clear that each nucleus can function as an independent oscillator, as ablation of one of them does not alter the circadian function (Van den Pol and Powley, 1979). Similarly, ablation of an entire nucleus, and components of the other, does not eliminate circadian functions until at least $75 \%$ of both nuclei are destroyed (Van den Pol and Powley, 1979; Mosko and Moore, 1979). Moreover, in vitro studies using hypothalamic SCN slices (usually about $400-500 \mu \mathrm{m}$, representing less than $50 \%$ of the total volume) show circadian oscillations of electrical activity and vasopressin release for several circadian cycles without apparent effect on the phase or period of the rhythm (Gillette, 1991; Bos and Mirmiran, 1990; Watanabe et al., 1993; Zhang and Aguilar-Roblero, 1995). Therefore, it is clear that there is redundancy in the SCN pacemaker.

The central issue that concerns us here is precisely this scale invariance, or the insensitivity of the period of the circadian rhythms to the number of cellular components of the regulating organ. On the one hand, one could mention a group of models with a small number of oscillators, but with complicated interconnections and feedbacks, tending to reproduce a spe- 
cific efferent behaviour of the clock (Carpenter and Grossberg, 1983). These models successfully reproduce several features of the circadian rhythms, like phase locking, phase response curves and resetting by light, but they do not address the important question of the origin of a circadian period. On the other hand, there is another group of mathematical models that are based on a network of many individual non-linear oscillators, which are able to obtain a circadian period from ultradian oscillators (Pavlidis, 1969). If the coupling is linear, then one can show quite generally that the output period of the system of coupled oscillators varies as the square root of the number of participating units. From this point of view, they cannot explain successfully the experimental data. In the present paper, we propose a new coupled-oscillator model that could explain the relative scale invariance of the circadian clock.

2. Theoretical Background-Large Systems of Coupled Oscillators. Our purpose is to demonstrate that a simple coupling of many ultradian oscillators (connected in the correct way) can exhibit circadian behaviour that is independent of the number of components for a sufficiently large number of components. If we succeed, this theory will give support to the hypothesis that biochemical ultradian oscillators are the origin of circadian rhythms.

The basic idea has been explored by Pavlidis (1969), who observed that a network of many coupled linear oscillators has a mode (minimum eigenvalue) of frequency very much smaller than the frequency of oscillation of each component. Consider a system of $N$ interconnected linear oscillators, $x_{k}, k=1, \ldots N$, satisfying

$$
\ddot{x}_{k}+\omega^{2} x_{k}-r \omega^{2} \sum_{\substack{i=1 \\ i \neq k}}^{N} x_{i}=0
$$

where there are elastic (linear) couplings of strength $r$ among all the identical oscillators of natural frequency $\omega$. The system has a single mode of oscillation at the low frequency

$$
\Omega=\omega \sqrt{(1-r(N-1))}
$$

for the sum

$$
s=\sum_{k=1}^{n} x_{k}
$$

This can be seen by summing up all equations (1), and the eigenvector is $(1,1, \ldots, 1)$. There is another $(N-1)$-degenerate mode at a high frequency $\alpha=\omega \sqrt{(1+r)}$, with $(N-1)$ linearly independent eigenvectors satisfying 
$\sum_{k} x_{k}=0$. Observe that if $r$ becomes too large $\left(\geqslant(N-1)^{-1}\right)$, then the system becomes unstable. The property displayed by equation (2) is retained by some non-linear systems used in biological modelling. For instance, consider a non-linear system of the type

$$
\frac{\partial^{2} \mathbf{x}}{\partial t^{2}}+\mathbf{F}(\mathbf{x})\left(\frac{\partial \mathbf{x}}{\partial t}\right)+\mathbf{A x}=0
$$

where $\mathbf{x}=\left(x_{1}, x_{2}, \ldots, x_{N}\right)^{T}$ and $\mathbf{A}$ is the matrix defining the elastic coupling. Where

$$
\sum_{j=1}^{n} F_{i j}(\mathbf{x})=f\left(\sum_{i=1}^{N} x_{i}\right)
$$

for all $i$, at least for the choice of an eigenvector column $(1,1, \ldots, 1)$. That is, the system has elastic and frictional couplings that depend only on the sum of the outputs:

$$
\ddot{x}_{k}+f\left(\sum_{i=1}^{n} x_{i}\right) \dot{x}_{k}+\omega^{2} x_{k}-r \omega^{2} \sum_{\substack{i=1 \\ i \neq k}}^{n} x_{i}=0 .
$$

It is clear that the final frequency will still be given by equation (2). A proper choice of $f$ will give a limit cycle with that frequency. Other extensions, considering only elastic coupling in equation (6) (that is, $f=$ $f\left(x_{k}\right)$ only) have been examined numerically by Pavlidis (1969), and a dependence of the frequency on the parameter $r(N-1)$ is still observed in the full non-linear model when $r$ is sufficiently large to induce mutual entrainment. This led us to think that equation (2) is quite general.

However, the behaviour of a general full non-linear system is impossible to describe. It could be argued that for biological systems it is very difficult to imagine a system of identical oscillators; therefore, it is useful to examine a system with a distribution of frequencies. This has been done numerically for a model of many simple limit-cycle oscillators (Matthews et al., 1991) considering a system with a general form for a supercritical Hopf bifurcation:

$$
\frac{d z_{j}}{d t}=\left(1-\left|z_{j}\right|^{2}+i \omega_{j}\right) z_{j}+\frac{r}{N} \sum_{i=1}^{N}\left(z_{i}-z_{j}\right)
$$

where $z_{j}$ is the position of the $j$ th oscillator in the complex plane and $\omega_{j}$ are distributed at random according to a symmetrical frequency distribution $g(\omega)$ around a mean $\bar{\omega}$. Among the diversity of complicated collective 
behaviours, there are situations, for strong coupling, in which large-amplitude oscillations arise with a frequency smaller than the width of the frequency distribution.

In any case, it seems that the problem is that the frequency of a collective mode in a large system of coupled oscillators goes to zero as $N \rightarrow \infty$. This actually contradicts the experimental findings that in the SCN the frequency of the circadian oscillation is practically independent of the number of neurons or glias. Here, one has two choices: either one denies that the circadian oscillation could be derived from ultradian oscillations (like the observed biochemical oscillations), or one proposes a system in which one is able to show a scale invariant frequency.

3. The Model. Suppose that we have a slightly non-linear system of the type of equation (6). This seems to be a very stringent condition, that we have to relax, but for the sake of simplicity, let us first consider this case. Let us change the connections to form an arbitrary network with elastic interactions between pairs. We know that the Hamiltonian of the linear system possesses a continuous symmetry, that is, it is invariant if one replaces $x_{k}$ by $x_{k}+\delta$. When this symmetry is broken by a mode of finite wavelength, one expects the presence of acoustic waves, whose frequency goes continuously to zero as the system becomes infinite. If the non-linearity is unable to change this property, one has to conclude that any arbitrary network of elastic springs has a low-frequency mode that approaches zero continuously as the size of the network increases. One way to get rid of this unwanted feature is to use a non-linear system of coupled pendulums (Winfree, 1967), or to capacitively couple limit-cycle oscillators (Othmer and Watanabe, 1992). Another way of stabilizing a low but non-zero frequency, without changing the elastic nature of the interaction, is to specify a hierarchical interconnection, that is, to consider that the oscillators are interconnected in different levels of organization.

Suppose that at the first level only a small number of ultradian oscillators with natural frequency $\omega_{0}$ are coupled elastically with strength $r=$ $r(0)$. Then, the system acts as a single oscillator with frequency $\omega_{1}=$ $\omega_{0} \sqrt{1-r(k-1)}$. Consider $k=2$, without loss of generality, as illustrated in Fig. 1. Then, one can couple these new oscillators with a constant $r(1)=f(r)$, and obtain a new oscillating system with frequency $\omega_{2}=$ $\omega_{1} \sqrt{1-r(1)}<\omega_{1}$. Continuing this process, one eventually obtains the final frequency

$$
\omega_{\nu}=\omega_{\nu-1} \sqrt{1-r(\nu)}
$$

where $N=2^{\nu}$, and $\nu$ labels the level of organization, or hierarchy. This recurrence relation can be written as a continued fraction that could 


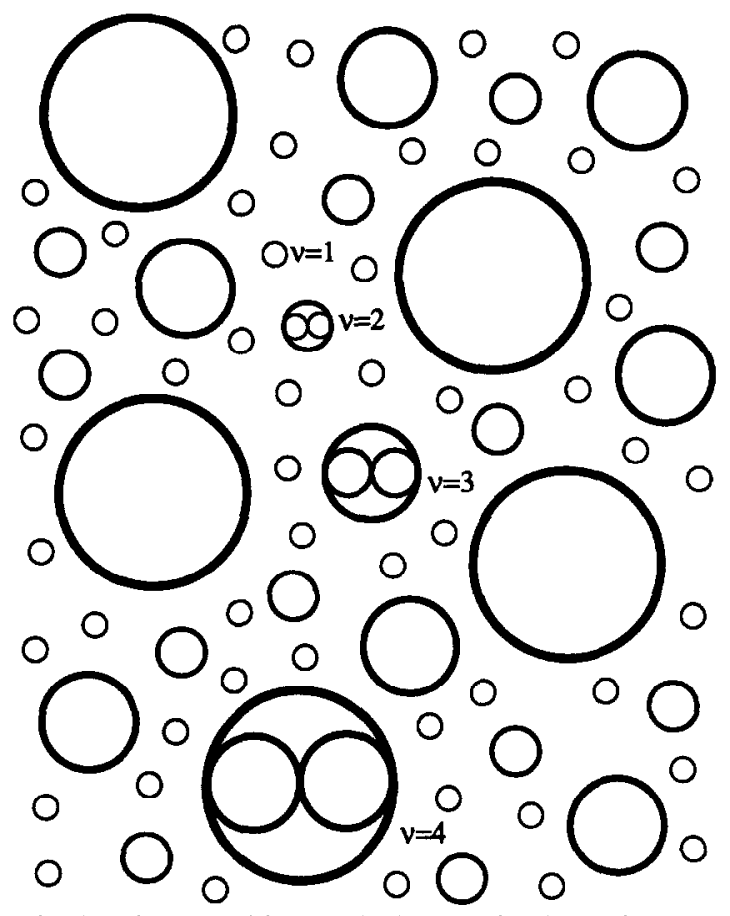

Figure 1. Sketch showing the hierarchical organization of coupled oscillators. The anatomical units are represented by small circles $(\nu=1)$. The bigger circles with heavier lines represent subsequent levels of organization $(\nu)$ of coupled oscillators. Although in this drawing only pairs of oscillators are coupled in each level, this is not necessarily true in reality, and it is not crucial for the model.

converge to a positive-definite number. As a simple example, suppose that the spring constant at a certain level is a fraction of the previous one, that is

$$
\begin{gathered}
r(\nu)=q r(\nu-1) \quad 0 \leqslant q \leqslant 1 ; \\
r(0)=r .
\end{gathered}
$$

In this case, the final frequency of the system of $N$ oscillators can be calculated exactly, and is given by

$$
\omega_{\nu}=\omega_{0} \sqrt{\prod_{i=0}^{\nu}\left(1-q^{i} r\right)}
$$

which has a limit in terms of $q$ and $r$. For the particular case when $r=q$, the analytical result can be found:

$$
\lim _{\nu \rightarrow \infty} \omega_{\nu}^{2}=\omega_{0}^{2}\left[\frac{1}{2} \vartheta_{2}(0) \vartheta_{3}(0) \vartheta_{4}(0)^{4}\right]^{1 / 6}
$$




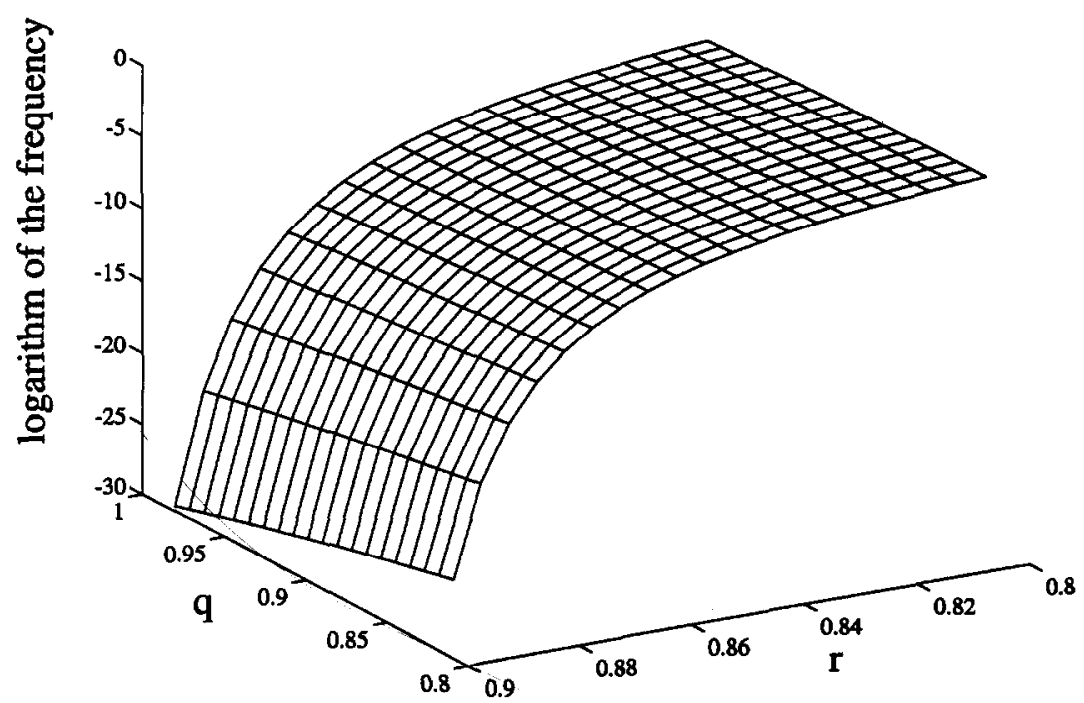

Figure 2. Final frequency taken from equation (10) for a region of the parameter space wherein a locked solution is found. Observe that the frequency could readily be reduced by a factor of $10^{-4}$, as needed for a circadian oscillation.

where $\vartheta_{i}(u)$ are the Elliptic Theta Functions (Gradshteyn and Ryzhik, 1980 ). For a general case, there is also a finite solution, except when $q=1$ (constant coupling at all levels), in which case $\omega_{\nu}$ goes exponentially to zero. This can be investigated numerically, as is shown in Fig. 2, where $\omega_{100}$ is plottcd in the $(q, r)$ space. Observe the logarithmic vertical scale, which shows that one can reduce the frequency four orders of magnitude easily with reasonable values of the parameters.

4. Numerical Calculations with a Simple Model. Let us now introduce a more realistic model to test the conclusions of the preceding arguments. Let us represent a single ultradian oscillator $i$ by a simple system with a limit cycle, the Van den Pol cquation

$$
\ddot{x}_{i}+\left(x_{i}^{2}-v\right) \dot{x}_{i}+\omega_{0}^{2} x_{i}=0
$$

which could be coupled to $k$ identical oscillators with elastic connections $r$ (in units of $\omega_{0}^{2}$ ), and written as

$$
\begin{gathered}
\dot{x}_{i}=\omega_{0} y_{i} \\
\dot{y}_{i}=-\omega_{0}\left(x_{i}-\sum_{j \neq i}^{k} r_{i j} x_{j}\right)+\left(v-x_{i}^{2}\right) y_{i}
\end{gathered}
$$

where $v>0$ is the bifurcation parameter. 
Let us build up a network with connections defined by a self-similar, hierarchical rule. The hierarchy is denoted by the index $\nu$. Then, the system is fractal and it is represented by

$$
\begin{gathered}
\dot{x}_{i}(\nu, t)=\omega_{\nu-1} y_{i}(\nu, t) \\
\dot{y}_{i}(\nu, t)=-\omega_{\nu-1}\left(x_{i}(\nu, t)-\sum_{j \neq i}^{k} r_{i j}(\nu, t) x_{j}(\nu, t)\right) \\
+\left(v-x_{i}^{2}(\nu, t)\right) y_{i}(\nu, t)
\end{gathered}
$$

where now, in order to mimic the actual $\mathrm{Ca}^{2+}$ oscillators and assuming that the coupling is only taking place if the Ca channels are open at the time when the wave arrives, we define

$$
r_{i j}(\nu, t)=\overline{r_{i j}}(\nu-1)\left[\tanh \left(\frac{\Delta_{i j}(\nu, t)}{a}\right)\right]
$$

where the bar denotes a time average. $\Delta_{i j}(\nu, t)=\left|\theta_{i}(t)-\theta_{j}(t)\right|$, with $\tan \left(\theta_{i}(t)\right)=y_{i}(\nu, t) / x_{i}(\nu, t)$, and $a$ the width of the switching. The output at level $\nu$ is taken to be

$$
s(\nu, t)=\frac{1}{k} \sum_{j=1}^{k} x_{j}(\nu, t) .
$$

The frequency at any level $\omega_{\nu}$ is calculated by taking the Fourier transform of the system output $s(\nu, \omega)$ :

$$
s\left(\nu, \omega_{\nu}\right)=\frac{1}{\Delta t} \int_{t}^{t+\Delta t} e^{-i \omega_{\nu} t} s(\nu, t) d t
$$

There are two parameters: $r$ and $a$. A large portion of the two-dimensional parameter space was explored using a simple improved Euler method, and it was found that the frequency stabilizes at a small value for some minimal $N$, provided $r$ is sufficiently large. In the case of $r=0.91$ and $a=0.34$, the final frequency $\omega=3.85 \times 10^{-4} \omega_{0}$ is attained for $N=8192$ oscillators and remains constant, within $0.1 \%$, for many more oscillators. This can simulate the actual biological system with the correct quantities (Van den Pol, 1980).

All calculations were made for $k=2$, but results can also be obtained for other values of $k$. A network of $N=2^{50}$ oscillators was examined more carefully using a Runge-Kutta method, and adding a random deviation to the frequencies of $\sim 10 \%$, following both a flat and a Gaussian distribu- 


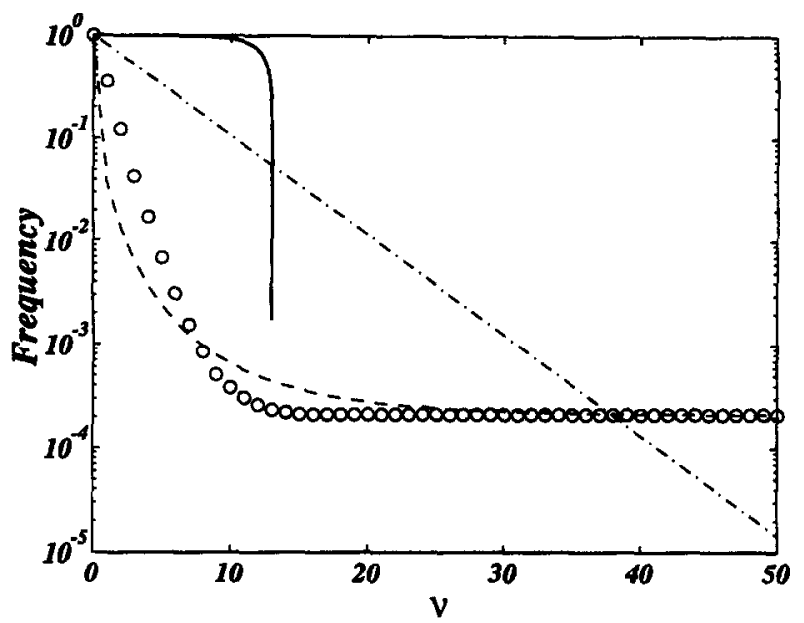

Figure 3. Final frequency of a hierarchical system of $N=2^{\nu}$ oscillators of fundamental frequency $\omega_{0}=1, a=0.34$ and $r=0.94$ (circles). The analytical result for the simpler fractal model with $q=0.9$ (broken line) also gives the same limiting value for large $\nu$, but is less effective in attaining stability. For comparison, the continuous line shows the dramatical square root dependence predicted by equation (2). The dash-dot line shows an exponential decay obtained with $q=1$.

tion. We verified that the frequency is stabilized for large $N$. Figure 3 illustrates a typical numerical calculation including noise; the values were chosen to give constant frequency for $N \geqslant 2^{12}=4096$ cells, and to obtain a reduction factor of $\sim 10^{-4}$, in order to represent a real clock. By allowing $k>2$, the hierarchical couplings could be less strong to obtain the same values.

5. Discussion. Here we discuss other possibilities of obtaining a robust clock without using a hierarchical network. It was pointed out a long time ago that some experimental results from the circadian pacemaker seemed to be in contradiction with the properties of models using limit-cycle oscillators (Winfree, 1967). Although it has been proved that these models can satisfactorily explain such experiments (Pavlidis, 1975, 1992), it is interesting to discuss the possibility of an array of pendulum-like oscillators since this system can present a cutoff frequency.

It is well known that a chain of pendulums coupled with springs presents a low cutoff frequency, which is independent of the number of oscillators. The problem with this system is that the low frequency is the same as for the normal mode of a single pendulum. This system is analogous to a high-pass electric filter.

One could obtain a lower value of the frequency by coupling the oscillators through a medium with high relative capacity. The possible 
realization of capacitively-coupled oscillators in biological systems has been discussed recently (Othmer and Watanabe, 1992), and it has been shown that one can obtain circadian periods from ultradian limit-cycle oscillators if this sort of coupling is strong. While the analysis is carried out for linear coupling, there are indications that similar behaviour is obtained for more general ways of coupling. At present, it is unclear if this model is more convenient than the one presented here, except that the latter does not depend on the nature of the limit-cycle function or on the way one couples the oscillators. In the analytical discussion, we presented a simple elastic coupling, but in the numerical calculation we used a more complicated coupling function, and the conclusions were unchanged (see Fig. 3).

It remains to be seen if the inclusion of non-linear (anharmonic) coupling results in a lower bound to the frequency and stabilization of the system with respect to the number of components. A general treatment is not possible, but we can gain further insight if we consider any oscillator still obeying equation (5), but with anharmonic coupling terms in $\mathbf{A}(\mathbf{x})$. These terms have to have only odd powers of $x$ since we want a bound state. A simplified version would be a sine function, for example. In that case, one obtains

$$
\ddot{s}+f(s) \dot{s}+\omega_{0}^{2} s-\omega_{0}^{2}(N-1) r \sum_{k=1}^{N} \sin \left(x_{k}\right)=0
$$

which, for the low-frequency mode $\left(x_{1}=x_{2}=\cdots=x_{N}\right)$, can be reduced to a form similar to equation (6). In the case of a simple pendulum, one arrives at a sine-Gordon equation, as in an array of Josephson junctions, and one expects the possibility of soliton solutions at finite frequency. These non-linear effects are expected to be present in a capacitively coupled network as well. The appearance of a lower limit for the frequency of this mode when one constructs a hierarchical array of oscillators with this kind of non-linear coupling still holds, which suggests that this feature is model independent, and one could use more sophisticated oscillators to be applied for specific biological modelling.

Welsh et al. (1995) observed circadian rhythms in the firing rate of individual SCN neurons living in a dissociated culture. They also observed that circadian oscillations from different neurons in the same culture were not synchronized. These observations seemed to be enough to conclude that SCN circadian pacemaker is formed from single cell circadian oscillators. However, a careful reading of the recent literature reveals that the question of the origin of circadian oscillations, either by coupled ultradian oscillators or by a collection of individual cells with circadian rhythms, is not settled yet. 
The acrophase of the circadian oscillations observed by Welsh et al. (1995) was related to the regular firing pattern. It is well known that this pattern is presented at all circadian times (Shibata et al., 1984; Thomson et al., 1984; Zhang et al., 1995). This means that if one could monitor the single-cell firing rate rhythms in SCN slice preparations, or even in vivo, for more than one day, they could be found not synchronized, although the clock would function normally.

Furthermore, Watanabe et al. (1993) measured vasopressin release in the same type of dissociated SCN culture, with neurons resting on a syncitium of astrocytes, and mentioned that in more than one hundred cultures a clear circadian oscillation of vasopressin was observed (monophasic oscillation), with the exception of few cultures in which plating cells were damaged by accident, or their density was very low. This seems to show that a minimum number of cells is needed for the clock to work properly, and supports the idea that coupled ultradian oscillators do play an important role in circadian clock generation.

Moreover, there is presently detailed quantitative evidence (Davis and Gorski, 1984) that the period of circadian rhythms is a function of the total volume of undamaged SCN. This phenomenon can be explained by the present model.

In the $\mathrm{SCN}, \mathrm{Ca}^{2+}$ currents are involved in the generation of action potentials and phase shifts in the circadian rhythms of electrical activity in vitro (Meijer and Rietveld, 1989; Aguilar-Roblero et al., 1996). Release of $\mathrm{Ca}^{2+}$ from intracellular deposits into the cytoplasm has been shown to be critical in the regulation of several neural processes (Aguilar-Roblero et al., 1996). The intracellular $\mathrm{Ca}^{2+}$ homeostasis has not been studied sufficiently in SCN. The present model is based on the intracellular $\mathrm{Ca}^{2+}$ ultradian oscillation in SCN glial cells (Van den Pol et al., 1992). The result of this heirarchical coupling leads to a stabilization of the output frequency of $\mathrm{Ca}^{2+}$ population oscillation to a circadian value that is robust. Recently, circadian oscillations of intracellular $\mathrm{Ca}^{2+}$ release channels, expressed by number of Ryanodine receptor, in SCN slice preparation were observed (Aguilar-Roblero et al., 1996). This is consistent with the output of our model.

The hierarchical coupling has to be dynamical, therefore any sigmoidal function of the type $f(x)=\tanh (x)$, assumed in the model, could mimic the dynamical state of the gap junction of astrocytes. It is well known that astrocytes are extensively coupled by gap junctions composed mainly of connexin 43 and can form a large syncitium. The permeability, and consequently the gap junction mediated communication, is regulated by particular conditions (Giaume and McCarthy, 1996; Verkhratsky and Kettenmann, 1996); although research in this area is still at its early stages, both theoretically and experimentally, and significant progress requires a transition from the understanding of basic properties to the study of function. On 
the other hand, preventing undesirable variations of the parameters of the ultradian oscillators from modifying the output circadian frequency, additional mechanisms would be required. These mechanisms should be ruled by the phylogeny and the ontogeny of the circadian function and are beyond the scope of this paper.

In all mathematical models of the biological clock, there are parameters that have to be used to make them fit the experiments quantitatively. In the present model, there are two basic parameters that fix the low-frequency solution of the equations. One could ask how, in reality, these parameters are determined. This question is, in general, far beyond the scope of mathematical models. However, the value of the low-level interaction $r=r(0)$ and the factor needed for frequency demultiplication could be encoded in the phylogeny and ontogeny of the circadian function.

6. Conclusions. One can summarize the results of the present study as follows:

1. This simple model shows that a hierarchical network of simple nonlinear oscillators presents scale invariance with respect to the number of components.

2. The stabilization of the frequency is very efficient, and with reasonable values of the parameters, one is able to lower the period four orders of magnitude, as needed for a circadian oscillation.

3. The model is general and may be easily elaborated to obtain more realistic properties of circadian rhythms, without modifying the scaleinvariance property.

4. The model is stable over a wide range of parameter values, and it is not crucial that these are not precisely defined, a very convenient property to model biological systems.

As we mentioned before, the use of multicomponent oscillator models has been successful in reproducing available experimental data. In any case, if one models the circadian rhythm with simple pendulums or limit-cycle oscillators, there remains the central question of obtaining a low-frequency oscillation that is stable and robust. As we have shown, the period of a set of coupled oscillators is a complicated function of the periods of the components, and under certain restrictions, one can still think of a long period behaviour. We discussed the case in which oscillators (harmonic or limit-cycle) are (elastically or non-linearly) coupled in a network, and we found that the frequency of the output is generally dependent on the number of oscillators. On the contrary, if one uses a hierarchical coupling, one can obtain a scale-invariant output frequency for sufficiently strong coupling that is practically model independent. 
We thank the Instituto de Física U.N.A.M. (P.K.M.), the Department of Theoretical Physics (R.A.B.) and the Centre for Mathematical Biology (L.Z.), Oxford University, for their hospitality during visits. We are indebted to M. F. Thorpe, L. Gonçalves H. Othmer, and R. Aguilar-Roblero for helpful discussions and comments on the manuscript. This work was supported by DGAPA-UNAM IN101894 (R.A.B.), by a Royal Society Fellowship (R.A.B.) and by a CONACYT postdoctoral fellowship (L.Z.). Part of this work was done while P.K.M. was on sabbatical at the Department of Mathematics, Williams College, Williamstown, MA, U.S.A.

\section{REFERENCES}

Aguilar-Roblero, R., J. L. Chávez and M. Díaz-Muñoz. 1996. Circadian modulation of intracellular $\mathrm{Ca}^{++}$release channel (Ryanodine receptor) in the suprachiasmatic nuclei. Fifth Meeting Soc. for Research on Biological Rhythms, Jacksonville, Florida, p. 63 (abstract).

Bos, N. P. A. and M. Mirmiran. 1990. Circadian rhythms in spontaneous neuronal discharges of the cultured suprachiasmatic nucleus. Brain Res. 511, 158-162.

Carpenter, G. A. and S. M. Grossberg. 1983. Mammalian circadian rhythms. In Oscillations in Mathematical Biology, J. P. E. Hodgson (Ed), pp. 102-196. Berlin: Springer-Verlag.

Davis, F. C. and R. A. Gorski. 1984. Unilateral lesions of the hamster suprachiasmatic nuclei: evidence for redundant control of circadian rhythms. J. Comp. Physiol. A 154, 221-232.

Drucker-Colín, R., R. Aguilar-Roblero, F. Garcia-Hernández, F. Fernández-Cancino and F. Bermúdez-Rattoni. 1984. Fetal suprachiasmatic nucleus transplants: diurnal rhythm recovery of lesioned rats. Brain Res. 311, 353-357.

Earnest, D. J., S. M. Digiorgio and C. D. Sladek. 1991. Effects of tetrodotoxin on the circadian pacemaker mechanism in suprachiasmatic explants in vitro. Brain Res. Bull. 26, $677-682$.

Edery, I., J. E. Rutila and M. Roshbach. 1994. Phase shifting of the circadian clock by induction of the Drosophila period protein. Science 237, 237-240.

Enright, J. T. 1980. Temporary precision in circadian systems: a reliable neuronal clock from unreliable components? Science 209, 1542-1545.

Gekakis, N., L. Saez, A.-M. Delahaye-Brown, M. P. Myers, A. Sehgal, M. W. Young, C. J. Weitz. 1995. Isolation of timeless by PER protein interaction: defective interaction between timeless protein and long-period mutant PER. Science 270, 811-815.

Giaume, C. and K. D. McCarthy. 1996. Control of gap-junctional communication in astrocytic networks. Trends Neurosci. 19, 319-325.

Gillette, M. U. 1991. SCN electrophysiology in vitro: rhythmic activity and endogenous clock properties. In Suprachiasmatic Nucleus, the Mind's Clock, D. C. Klein, R. Y. Moore and S. M. Reppert (Eds), ch. 6, pp. 125-143. New York: Oxford University Press.

Gradshteyn, I. S. and I. M. Ryzhik. 1980. Table of Integrals, Series, and Products, ch. 8.17, pp. 921-925. New York: Academic Press.

Hardin, P. E., J. C. Hall and M. Rosbach. 1990. Feedback of the Drosophila period gene product on circadian cycling of its messenger RNA levels. Nature 343, 536-540.

Inouye, S. I. T. and H. Kawamura. 1979. Persistence of circadian rhythmicity in the mammalian hypothalamic "island" containing the suprachiasmatic nucleus. Proc. Natl. Acad. Sci. USA 76, 5962-5966.

Konopka, R. J. and S. Benzer. 1971. Clock mutants of Drosophila melanogaster. Proc. Natl. Acad. Sci. 68, 2112.

Lee, C., V. Vaishali, T. Itsukaichi, K. Bae and I. Edery. 1996. Resetting the Drosophila clock by photic regulation of PER and a PER-TIM complex. Science 271, 1740-1741. 
Lehman, M. N., R. Silver, W. R. Gladstone, R. M. Kahn, M. Gibson and E. L. Bittman. 1987. Circadian rhythmicity restored by neural trasplant. Immunocytochemical characterization of the graft and its interaction with host brain. J. Neurosci. 7, 1626-1638.

Matthews, P. C., R. E. Mirollo and S. H. Strogatz. 1991. Dynamics of a large system of coupled nonlinear oscillators. Physica D 52, 293-331.

Meijer, J. H. and W. J. Rietveld. 1989. Neurophysiology of the suprachiasmatic circadian pacemaker in rodents. Physiol. Rev. 69, 671-707.

Moore, R. Y. 1992. The suprachiasmatic nucleus and the circadian timing system. In Circadian Rhythms, Discussions in Neuroscience, P. J. Magistratti (Ed), Vol. 8, Nos. 2-3, ch. 5, pp. 26-33. Amsterdam: Elsevier Science Publishers B.V.

Mosko, S. S. and R. Y. Moore. 1979. Neonatal suprachiasmatic nucleus lesions. Brain Res. 164, 17-38.

Myers, M. P., K. Wagner-Smith, A. Rothenfluh-Hilfiker and M. W. Young. 1996. Lightinduced degradation of the Drosophila circadian clock. Science 271, 1736-1740.

Newman, G. C., F. E. Hospod, C. S. Patlak and R. Y. Moore. 1992. Analysis of in vitro glucose utilization in a circadian pacemaker model. J. Neurosci. 12, 2015-2021.

Othmer, H. G. and M. Watanabe. 1992. Novel mechanism for generating circadian periods from fast oscillators. In Abst. of the 2nd Annual Conf. of the Japan Soc. for Industrial and Applied Math., pp. 1-2.

Pavlidis, T. 1969. Populations of interacting oscillators and circadian rhythms. J. Theor. Biol. 22, 418-436.

Pavlidis, T. 1975. Spatial organization of chemical oscillators via an averaging operator. J. Chem. Phys. 63, 5269-5273.

Pavlidis, T. 1992. Mathematical models. In Handbook of Behavioral Neurobiology, J. F. Aschoff (Ed), Vol. 4, ch. 4, pp. 41-54. New York: Plenum Press.

Pittendrigh, C. S. 1974. Circadian organization in cells and the circadian organization of the multicellular system. In Neurosciences Third Study Program, S. O. Schmitt and F. G. Worden (Eds). Cambridge, MA: MIT Press.

Prosser, R. E., J. D. Miller and H. C. Heller. 1990. Sertonin agonist phase-shifts in circadian clock in the suprachiasmatic nuclei in vitro. Brain Res. 534, 336-339.

Ralph, M. R., R. G. Foster, F. D. Davis and M. Menaker. 1990. Transplanted suprachiasmatic nucleus determines circadian period. Science Wash. DC 247, 975-978.

Schwartz, W. J., R. A. Gross and M. T. Morton. 1987. The suprachiasmatic nuclei contain a tetrodotoxin-resistant circadian pacemaker. Proc. Natl. Acad. Sci. USA 84, 1694-1698.

Sehgal, A., J. L. Price, B. Mam and M. W. Young. 1994. Loss of circadian behavioral rhythms and per RNA oscillation in the Drosophila mutant timeless. Science 263, 1603.

Sehgal, A., A Rothenfluh-Hilfiker, M. Hunter-Ensor, Y. Chen, M. P. Myers and M. W. Young. 1995. Rhythmic expression of timeless: a basis for promoting circadian cycles in period gene autoregulation. Science 270, 808-810.

Shibata, S., Y. Oomura, S. Y. Liou and S. Ueki. 1984. Electrophysiological studies of the development of suprachiasmatic neuronal activities in hypothalamic slice preparation. Brain Res. 13, 29-35.

Thomson, A. M., D. C. West and I. G. Vlachonikolis. 1984. Regular firing of suprachiasmatic neurons maintained in vitro. Neurosci Lett. 52, 329-334.

Van den Pol, A. N. and T. P. Powley. 1979. A fine grained anatomical analysis of the role of the rat suprachiasmatic nucleus in circadian rhythms of feeding and drinking. Brain Res. 160, 307-326.

Van den Pol, A. N. 1980. The hypothalamic suprachiasmatic nucleus of rat: intrinsic anatomy. J. Comp. Neurol. 191, 661-702.

Van den Pol, A. N., S. M. Pinkbeiner and A. H. Cornell-Bell. 1992. Calcium excitability and oscillations in suprachiasmatic nucleus neurons and glia in vitro. J. Neurosci. 12, 2649-2664.

Verkhratsky, A. and H. Kettenmann. 1996. Calcium signalling in glial cells. Trends Neurosci. 19, 346-352.

Vosshall, L. B., J. L. Price, A. Sehgal, L. Saez and M. W. Young. 1994. Block in nuclear localization of period protein by a second clock mutation, timeless. Science 263, 1606. 
Watanabe, K., N. Koibuchi, H. Ohtake and S. Yamaoka. 1993. Circadian rhythms of vasopressin release in primary cultures of rat suprachiasmatic nucleus. Brain Res. 624, 115-120.

Welsh, D. K., D. E. Logothetis, M. Meister and S. Reppert. 1995. Individual neurons dissociated from rat suprachiasmatic nucleus express independently phased circadian firing rhythms. Neuron 14, 697-706.

Winfree, A. T. 1967. Biological rhythms and the behavior of population of coupled oscillators. J. Theor. Biol. 16, 15-42.

Winfree, A. T. 1975. Unclocklike behavior of biological clocks. Nature 253, 315-319.

Zhiang, L. and R. Aguilar-Roblero. 1995. Asymmetrical electrical activity between the suprachiasmatic nuclei in vitro. NeuroReport 6, 537-540.

Zhang, L., R. Aguilar-Roblero, R. A. Barrio and P. K. Maini. 1995. Rhythmic firing patterns in suprachiasmatic nucleus (SCN): the rôle of circuit interactions. Int. J. Bio-med. Comp. 38, 23-31.

Received 21 January 1996

Revised version accepted 8 October 1996 